\title{
Synovial fluid chondroitin and keratan sulphate epitopes, glycosaminoglycans, and hyaluronan in arthritic and normal knees
}

\author{
Carolyn Belcher, Reehana Yaqub, Fiona Fawthrop, Michael Bayliss, Michael Doherty
}

\begin{abstract}
Objectives-To determine concentrations of chondroitin sulphate (CS) and keratan sulphate (KS) epitopes, glycosaminoglycans (GAGs) and hyaluronan (HA) in knee synovial fluid (SF) from normal subjects and patients with osteoarthritis (OA) or rheumatoid arthritis (RA), to test whether these variables may be used as markers of the OA process.

Methods-OA was subdivided into large joint OA (LJOA), nodal generalised OA (NGOA), and OA with calcium pyrophosphate crystal deposition (CPA). Clinical assessment of inflammation (0-6) was undertaken on $O A$ and RA knees. Knee SF was examined by enzyme linked immunosorbent assay for: CS epitopes, using monoclonal antibodies 3-B-3 and 7-D-4; KS epitope using monoclonal antibody 5-D-4; and HA, using biotinylated HA binding region of cartilage proteoglycan. Total sulphated GAGs were measured by dye binding with 1:9 dimethylmethylene blue.
\end{abstract}

Results-Increased SF 3-B-3 concentrations and 3-B-3/GAG ratio were found in OA, compared with RA or normal knees, with higher 3-B-3 and 3-B-3/GAG in LJOA and NGOA than in CPA. SF 7-D-4 and 7-D-4/GAG were reduced in RA, compared with normal and OA; SF 5-D-4 was reduced in OA compared with normal. GAG and HA concentrations were decreased in both OA and RA. No correlations with radiographic scores were observed, but SF 7-D-4 was lower in 'inflamed' compared with 'non-inflamed' $R A$ and $O A$ knees. In patients with bilateral samples there were strong correlations between right and left knees for all SF variables.

Conclusions-Changed concentrations of SF CS and KS can be detected in OA with a profile that differs from that seen in RA. Clinical subgrouping and local joint inflammation may influence these measures, supporting different pathogenesis within OA subgroups and requirement for careful patient characterisation in SF studies.

(Ann Rheum Dis 1997;56:299-307)

Articular cartilage is the main site of irreparable damage in osteoarthritis (OA). ${ }^{1}$ Breakdown products of cartilage catabolism have therefore been studied as potential markers of the events that lead to cartilage attrition. The most abundant cartilage proteoglycan is aggrecan, comprising a protein core to which are attached many chondroitin sulphate (CS) and keratan sulphate (KS) chains. In health aggrecan binds to link protein and hyaluronan (HA) to form macromolecules immobilised within the intact collagen matrix.

Monoclonal antibodies have been produced with specificities against structural carbohydrate epitopes on the KS and CS side chains. The antibody 5-D-4 recognises 'oversulphated' domains in the heptasaccharide of $\mathrm{KS}^{2}$ and has been used to estimate $\mathrm{KS}$ in body fluids, principally serum..$^{3-5}$ Although high serum concentrations of $\mathrm{KS}$ are reported in patients with 'hypertrophic' hip OA, ${ }^{6}$ other studies of patients with generalised or large joint OA have not found high concentrations. ${ }^{57}$ Although serum KS can reflect acute major loss of cartilage in experimental $\mathrm{OA}^{8}$ and is often regarded as a marker of cartilage turnover, its value in assessing activity ${ }^{57}$ or prognosis $^{9}$ of OA remain in question. However, the much higher levels of KS in synovial fluid (SF) from OA knees, and the lack of correlation with serum levels ${ }^{5}$ suggest that SF levels of KS may prove a more sensitive indicator of local joint processes.

Certain neo-epitopes on CS chains, recognised by the monoclonal antibodies 3-B-3 and 7-D-4, may also reflect aspects of the OA process. These epitopes are absent or only weakly expressed in adult canine cartilage, but occur in very high levels in experimental OA cartilage. ${ }^{1011}$ Increased levels also occur in human articular cartilage from OA knees ${ }^{12}$ and in SF from knees with traumatic meniscal or cruciate ligament tears..$^{13}$ It has been postulated that the increase in these epitopes may reflect the early cartilage response to insult in which chondrocytes re-initiate a high level of matrix synthesis comparable to that seen during development. ${ }^{13}$

$\mathrm{OA}$ is a heterogeneous condition and a number of 'subsets' with possible differing pathogenesis have been suggested. In this study we have compared knee SF levels of KS and CS epitopes, HA and total GAGs from (1) patients with OA, characterised with respect to defined clinical subsets, (2) patients with knees affected by rheumatoid arthritis (RA) (a joint disease comparator), and (3) subjects with asymptomatic, normal knees (normal controls). The objective was to test whether these 
variables may be used firstly as a marker for the OA process, and secondly to distinguish between different subsets of OA.

\section{Methods}

Local Research Ethics Committee approval was obtained for this study.

PATIENT CHARACTERISATION

Hospital referred patients with symptomatic knees affected by OA or RA were studied. Patients with knee OA all had radiographic evidence of joint space narrowing and osteophyte in one or more knee compartments (medial or lateral tibiofemoral, patellofemoral). Other joint abnormality was excluded on the basis of full clinical assessment, radiographic features, SF examination, and serological and biochemical tests. ${ }^{14}$ The RA patients all fulfilled ACR criteria for definite $\mathrm{RA}^{15}$ and all had symptoms and signs of knee involvement by RA.

In each $\mathrm{OA}$ and $\mathrm{RA}$ patient standardised radiographs of both knees were taken; standing, knee extended, anteroposterior views (tibiofemoral compartments), plus lateral $30^{\circ}$ flexion views (patellofemoral compartments). Radiographic severity of OA at each knee compartment was graded according to a modified Kellgren and Lawrence system ${ }^{16}$ by one observer. For each of the three compartments a score was given for narrowing (0-3) and osteophyte (0-3); summated scores for each knee were then calculated for each feature (0-9). The total, global score for each knee was the sum of the two summated scores for narrowing and osteophyte (0-18). To assess intraobserver variability 80 films were re-examined on two separate occasions. Hand radiographs were undertaken on all subjects with OA and examined for evidence of definite narrowing and definite osteophyte (present/absent) in proximal and distal interphalangeal joints.

The knee OA patients were subdivided into three subsets: (1) Large joint OA (LJOA): knee $\mathrm{OA} \pm \mathrm{OA}$ of other large joints (hips/shoulders) but no Heberden's nodes or clinical/ radiographic evidence of polyarticular hand interphalangeal OA. (2) Nodal generalised OA (NGOA): knee OA, plus Heberden's ( \pm Bouchard's) nodes and $x$ ray evidence of interphalangeal OA (narrowing and/or osteophyte) affecting two or more rays of each hand. (3) Chronic pyrophosphate arthropathy (CPA): knee OA with calcium pyrophosphate dihydrate crystals identified in their knee SF $( \pm$ radiographic chondrocalcinosis). Crystal identification was by compensated polarised light microscopy of fresh SF. Patients that overlapped these groups, for example those with NGOA and SF pyrophosphate crystals, were not included in the study.

For OA and RA knees clinical inflammation at time of aspiration was designated 'active' (score 4-6) or 'inactive' (score 0-2) using a summated score of six clinical parametersincreased warmth, effusion, anterior joint-line synovial thickening, joint-line tenderness, early morning stiffness, inactivity stiffness. ${ }^{17}$ Knees scoring 3 were excluded. The components of this system show good interobserver and intraobserver reproducibility. ${ }^{18}$

Knee SF was also obtained from healthy volunteers (sufficient SF obtained from $80 \%$ of volunteers), as previously described. ${ }^{19}$ Normal knees were defined as those that had never had symptoms (pain, stiffness, swelling) and that were normal on examination, in a subject with no clinical evidence of arthropathy at other sites. Knee radiographs (standing anteroposterior and lateral $30^{\circ}$ flexion views) were obtained on subjects aged over 50 years to exclude occult pathology (for example, minor OA, chondrocalcinosis).

SAMPLE COLLECTION AND PRETREATMENT

Knees were aspirated to apparent dryness via a medial approach, and the SF volume recorded. A small sample of fresh SF was examined for crystals and the remainder collected into sterile plastic containers on ice, centrifuged at $2500 \mathrm{~g}$ for 15 minutes at $4^{\circ} \mathrm{C}$, and the supernatant stored at $-80^{\circ} \mathrm{C}$.

SF samples were treated with 0.5 units hyaluronidase (Streptomyces hyalurolyticus) per $100 \mu \mathrm{l}$ fluid for 30 minutes at room temperature. Samples for $\mathrm{HA}$ and $\mathrm{GAG}$ estimation were papain digested ${ }^{13}: 20 \mu$ papain suspension (type III) was added to $1 \mathrm{ml}$ buffer containing $10 \mathrm{mM}$ sodium EDTA, $20 \mathrm{mM}$ cysteine (added immediately before use) and $0.4 \mathrm{M}$ sodium acetate, $\mathrm{pH}$ 5.8. To $100 \mu \mathrm{l}$ of this solution was added $100 \mu \mathrm{SF}$ and incubated overnight at $60^{\circ} \mathrm{C}$. The enzyme was inactivated by addition of iodoacetic acid to a concentration of $4 \mathrm{mM}$, then the samples were mixed and centrifuged at $12000 \mathrm{~g}$ for four minutes. ${ }^{13}$

ELISA ESTIMATIONS

Antibodies 3-B-3 and 7-D-4 were a generous donation from Professor B Caterson, University of Wales, Cardiff. The 3-B-3 assay was modified from the procedure described by Ratcliffe et al. ${ }^{1320}$ Microtitre plate wells were coated with $200 \mu \mathrm{l}$ of $25 \mathrm{ng} / \mathrm{ml}$ chondroitinase ABC digested pig laryngeal cartilage proteoglycan (PLCP) freshly prepared in 20 $\mathrm{mM}$ sodium carbonate buffer ( $\mathrm{pH}$ 9.6), incubated overnight at room temperature, then washed four times with TRIS incubation buffer $(0.1 \%$ bovine serum albumin (BSA), $0.1 \%$ nonidet NP40, $0.01 \mathrm{M}$ TRIS $\mathrm{HCl}, 0.15$ $\mathrm{M} \mathrm{NaCl}$ ). Plates were blocked with $1 \% \mathrm{BSA}$ in TRIS incubation buffer at $37^{\circ} \mathrm{C}$ for two hours. After washing with TRIS, $100 \mu \mathrm{l}$ of competitor (test sample or standard) was added to the wells. Standards were prepared from PLCP in the range 3.9-500 $\mathrm{ng} / \mathrm{ml}$. SF samples were treated with hyaluronidase as above. A maximum binding well was prepared containing no competitor. Some $100 \mu \mathrm{l}$ of 3-B-3 antibody (1:10 000) was added and the plates incubated for two hours at $37^{\circ} \mathrm{C}$. After washing with TRIS, $200 \mu \mathrm{l}$ of goat antimouse IgM conjugated to horseradish peroxidase (1:4000) was added to each well and incubated at $37^{\circ} \mathrm{C}$ for $1-1.5$ hours. After washing with TRIS, $200 \mu$ peroxidase substrate (ABTS) was added and incubated at $37^{\circ} \mathrm{C}$ for $20-30$ 
Table 1 Patient and normal subject characteristics

\begin{tabular}{lllllll}
\hline Subject group & $\begin{array}{l}\text { Number of } \\
\text { subjects }\end{array}$ & Male:female & $\begin{array}{l}\text { Age (mean } \\
\text { (range) years) }\end{array}$ & $\begin{array}{l}\text { Osteophyte formation } \\
\text { (median (IQ range)) }\end{array}$ & $\begin{array}{l}\text { foint space } \\
\text { narrowing (median } \\
\text { (IQ range) }\end{array}$ & $\begin{array}{l}\text { Total } \mathrm{x} \text { ray score } \\
\text { (median (IQ } \\
\text { range) }\end{array}$ \\
\hline Normal & 38 & $13: 25=1: 1.92$ & $45(21-83)$ & - & - & - \\
RA & 29 & $6: 23=1: 3.83$ & $59(28-85)$ & $1(0-3)$ & $3.5(2-5.5)$ & $5(3-8)$ \\
LJOA & 57 & $26: 31=1: 1.19$ & $69(41-90)$ & $3(2-5)$ & $3(2-4)$ & $6(4-8)$ \\
NGOA & 22 & $2: 20=1: 10$ & $72(56-87)$ & $4(3-6)$ & $4(3-4)$ & $8(6-10)$ \\
CPA & 23 & $13: 10=1.3: 1$ & $70(49-92)$ & $4(3-8.5)$ & $4(3-5)$ & $8.5(6.5-13)$ \\
\hline
\end{tabular}

minutes. Absorbances were read at $405 \mathrm{~nm}$ on a Dynatech MR5000 plate reader, using BioLinx software. The ratio of absorbances to the absorbance of the maximum binding well was calculated for all standards and samples. A standard curve was plotted of ratio against standard concentration and used to determine concentrations of samples.

The 7-D-4 ELISA was carried out in a similar way. Wells were coated with $200 \mu \mathrm{l}$ of $3 \mu \mathrm{g}$ dye binding $/ \mathrm{ml}$ bovine tracheal cartilage proteoglycan (BTCP) freshly prepared in $20 \mathrm{mM}$ sodium carbonate $(\mathrm{pH}$ 9.6). Standards were prepared from BTCP in the range 0.0234-6 $\mu \mathrm{g} / \mathrm{ml}$. 7-D-4 antibody was added at a dilution of 1 in 25000 .

The immunoassay for 5-D-4 was a modification of the procedure described by Thonar et al. ${ }^{313}$ Microtitre plate wells were



Figure 1 Correlation between measurement of 3-B-3 in left and right knees of individual patients. When tested by Spearman's rank correlation, $\mathrm{r}=0.7324, p<0.001$. coated with $200 \mu \mathrm{l}$ of $50 \mathrm{ng} / \mathrm{ml}$ PLCP freshly prepared in $20 \mathrm{mM}$ sodium carbonate buffer $(\mathrm{pH}$ 9.6). Plates were incubated at room temperature for two hours, then overnight at $4^{\circ} \mathrm{C}$. On a separate plate $125 \mu \mathrm{l}$ of competitor (test sample or standard) was added to the wells. Standards were prepared from PLCP in the range $0.78-200 \mathrm{ng} / \mathrm{ml}$. SF samples were treated with hyaluronidase as above. A maximum binding well was prepared containing no competitor. Some $125 \mu \mathrm{l}$ of 5D4 antibody (1:70 000) was added to all wells except blanks and plates were incubated for one hour at room temperature, then at $4^{\circ} \mathrm{C}$ overnight. Coated plates were washed with PBS/TWEEN (0.123 M NaCl, $0.01 \quad \mathrm{M}$ $\mathrm{Na}_{2} \mathrm{HPO}_{4}, 3.16 \mathrm{mM} \mathrm{KH}_{2} \mathrm{PO}_{4}, 0.05 \%$ TWEEN $20, \mathrm{pH}$ 5.3). Competitor/antibody mixture $(200 \mu \mathrm{l})$ was transferred from inhibition plate to coated plate and incubated for one hour at $37^{\circ} \mathrm{C}$. After washing with PBS/TWEEN, 200 $\mu l$ of goat antimouse IgG conjugated to horseradish peroxidase $(1: 25000)$ was added to each well and incubated for one hour at $37^{\circ} \mathrm{C}$. After washing with PBS/TWEEN, $200 \mu$ l substrate (ABTS) was added and incubated at $37^{\circ} \mathrm{C}$ for $15-30$ minutes. Absorbances were read at $405 \mathrm{~nm}$ and results calculated as above.

The assay of HA was a modification of the procedure described by Fosang et al. ${ }^{21}$ Samples were papain digested before testing. Microtitre plate wells were coated with $200 \mu \mathrm{l}$ of $25 \mu \mathrm{g} / \mathrm{ml}$ HA freshly prepared in $20 \mathrm{mM}$ sodium carbonate buffer ( $\mathrm{pH}$ 9.6). Plates were incubated overnight at $4^{\circ} \mathrm{C}$, washed with PBS/ TWEEN (0.123 M NaCl, $0.01 \mathrm{M} \mathrm{Na}_{2} \mathrm{HPO}_{4}$, $0.05 \%$ TWEEN 20, pH 7.2), then blocked with $1 \%$ BSA in PBS/TWEEN for two hours at $37^{\circ} \mathrm{C}$. After washing with PBS/TWEEN, 100 $\mu \mathrm{l}$ of competitor (test sample or standard) was added to the wells. Standards were prepared from $\mathrm{HA}$ in the range $0.0195-5 \mathrm{ng} / \mathrm{ml}$. A maximum binding well was prepared containing no competitor. One hundred $\mu \mathrm{l}$ of biotinylated binding region (BBR) was added to all wells except blanks, and plates were incubated overnight at room temperature. After washing with PBS/TWEEN, $200 \mu \mathrm{l}$ of streptavidinbiotinylated horseradish peroxidase complex (1:3000) was added to each well, and incubated at $37^{\circ} \mathrm{C}$ for 30 minutes. After washing with PBS/TWEEN, $200 \mu$ l substrate (ABTS) was added and incubated at $37^{\circ} \mathrm{C}$ for 20-30 minutes. Absorbances were read at 405 $\mathrm{nm}$ and results calculated as above.

DIMETHYLMETHYLENE BLUE ASSAY OF TOTAL SULPHATED GAGS

The method used was modified from the procedure described by Farndale et al. ${ }^{223}$ 
Table 2 Correlations between right and left knees for all parameters

\begin{tabular}{llll}
\hline & Number & Correlation coefficient & $p$ Value \\
\hline Normal & 11 & 0.75 & \\
5D4 & 12 & 0.72 & 0.0054 \\
3B3 & 7 & 0 & 0.0064 \\
7D4 & 9 & 0.73 & NS \\
HA & 6 & 0.66 & 0.0219 \\
GAG & 5 & 0.6 & NS \\
5D4/GAG & 6 & 0.76 & NS \\
3B3/GAG & 3 & Unable to calculate & Unable to calculate \\
7D4/GAG & & & \\
OA & 34 & 0.45 & 0.0075 \\
5D4 & 45 & 0.72 & 0.0000 \\
3B3 & 35 & 0.67 & 0.0000 \\
7D4 & 32 & 0.40 & 0.0211 \\
HA & 37 & 0.29 & NS \\
GAG & 27 & 0.39 & 0.0431 \\
5D4/GAG & 34 & 0.67 & 0.0000 \\
3B3/GAG & 28 & 0.69 & 0.0000 \\
7D4/GAG & & & \\
RA & 10 & 0.53 & NS \\
5D4 & 8 & 0.40 & NS \\
3B3 & 10 & 0.84 & 0.0011 \\
7D4 & 10 & 0.26 & NS \\
HA & 8 & 0.21 & NS \\
GAG & 7 & 0.21 & NS \\
5D4/GAG & 4 & -0.2 & NS \\
3B3/GAG & 7 & 0.68 & NS \\
7D4/GAG & & & \\
\hline
\end{tabular}

Samples were papain digested and the supernatants treated with hyaluronidase. A working solution of dye was prepared by mixing $20 \mathrm{mg} 1,9$ dimethylmethylene blue, 5 $\mathrm{ml}$ ethanol, and one litre formate buffer $(0.1$ $\mathrm{M}, \mathrm{pH}$ 3.5). Standards were prepared from CS in the range $5-90 \mu \mathrm{g} / \mathrm{ml}$. Forty $\mu$ l of standard or sample was added to $250 \mu \mathrm{l}$ dye in a microtitre plate. Absorbances were read at $570 \mathrm{~nm}$ after two minutes on a Dynatech MR5000 plate reader, using BioLinx software.

STATISTICAL ANALYSIS

3-B-3, 7-D-4 and 5-D-4 were expressed as both concentrations and as ratios to total GAG concentrations. Multiple regression analysis was undertaken for comparisons between disease groups (OA, RA, normal) to account for the confounding factor of age. Comparisons within disease subgroups of the same age (NGOA, LJOA and CPA; active versus inactive within OA and RA) was by Friedman two way analysis of variance and the Mann-Whitney U test with a Bonferroni correction. Correlations were calculated using Spearman's correlation coefficient.

\section{Results}

Table 1 shows the demographic details. There was definite dependence between age and disease status $(p<0.001)$. Both the $R A$ and normal groups were younger than the OA group ( $\mathrm{p}<0.05, \mathrm{p}<0.01$ respectively) and the normal group was younger than the RA group $(p=0.05)$. There was no significant difference in total $x$ ray scores or narrowing between the disease groups. As expected, the RA group had a lower osteophyte score than all $\mathrm{OA}$ subgroups (LJOA, $p=0.03$; NGOA, $p=0.01$; CPA, $\mathrm{p}=0.01)$. In OA subjects with bilateral SF samples $(n=39)$, there was correlation between right and left knee $x$ ray scores ( $r$ values $0.77,0.81$, and 0.84 for osteophyte, narrowing, and summated scores respectively; all $\mathrm{p}<0.0001)$. There were only $10 \mathrm{RA}$ subjects with paired samples; $x$ ray correlations in this small group did not reach significance. Low intraobserver variability for grading of knee radiographs was demonstrated (global knee score $\kappa=0.75$, agreement $=0.88$, variance $=0.005)$. For the SF measurements, intra-assay variations ranged from $6-13 \%$ and inter-assay variations were $12 \%$ (3-B-3, 7-D-4), $14 \%$ (7-D-4), 20\% (HA), and 28\% (GAG).

CORRELATION BETWEEN RIGHT AND LEFT KNEES FOR SF VARIABLES

For all subjects with SF data on right and left knees there was strong correlation between knees of a single individual for 3-B-3 $(r=0.73$; fig 1) and all other assays ( $r$ values ranging from 0.51-0.74; all $\mathrm{p}<0.001)$. Division of paired samples according to diagnosis resulted in small numbers in each category except for OA (table 2), in which all correlations between knees remained except for GAG. Despite small numbers, three correlations remained in the normal SF group (5-D-4, 3-B-3, and HA). Because of these correlations the data could not be treated as independent and only one knee (right or left) per person was allocated at random for between group analysis.

CHANGES WITH AGE IN NORMAL KNEE SF

In normal subjects SF 3-B-3 concentrations showed a positive correlation with age $(r=$ $0.49, \mathrm{p}<0.05)$, whereas hyaluronan showed a negative correlation with age $(r=-0.71$, $\mathrm{p}<0.01)$. No other age associations were observed for other SF measurements.

COMPARISON BETWEEN RA, OA, AND NORMAL

KNEES FOR SF VARIABLES

Figure 2 shows the results expressed as concentrations. Using multiple regression to account for the confounding factor of age, 3-B-3 concentrations were increased in OA compared with RA and normal knees $(p<0.001, p<0.01$ respectively), whereas concentrations in RA were not different from normal controls. 7-D-4 levels were lower in RA compared with $\mathrm{OA}$ and normal knees (both $\mathrm{p}<0.001$ ) with no difference between OA and normal. 5-D-4 levels were lower in OA than normal knees $(p<0.01)$, but RA levels were not significantly different from those in OA or normal knees. Compared with normal knees, HA concentrations were reduced in both $\mathrm{OA}$ and RA $(p<0.01)$ with no difference between the two disease groups. Total GAG levels were similarly lower in both OA and RA compared with normal $(p<0.05, p<0.01$ respectively) with no difference between OA and RA.

Figure 3 shows the results expressed as ratios of $\mathrm{CS}$ or $\mathrm{KS}$ epitope to total GAG concentrations. The 3-B-3/GAG ratio was increased in OA compared with normal $(\mathrm{p}<0.01)$, with no differences between RA and normal, or between RA and OA. The $7-\mathrm{D}-4 / \mathrm{GAG}$ ratio was reduced in $\mathrm{RA}$ compared with OA and normal $(p<0.001$ and $\mathrm{p}<0.01$ respectively) with no difference between $\mathrm{OA}$ and normal knees. There were no significant differences between groups for 5D4/GAG. 


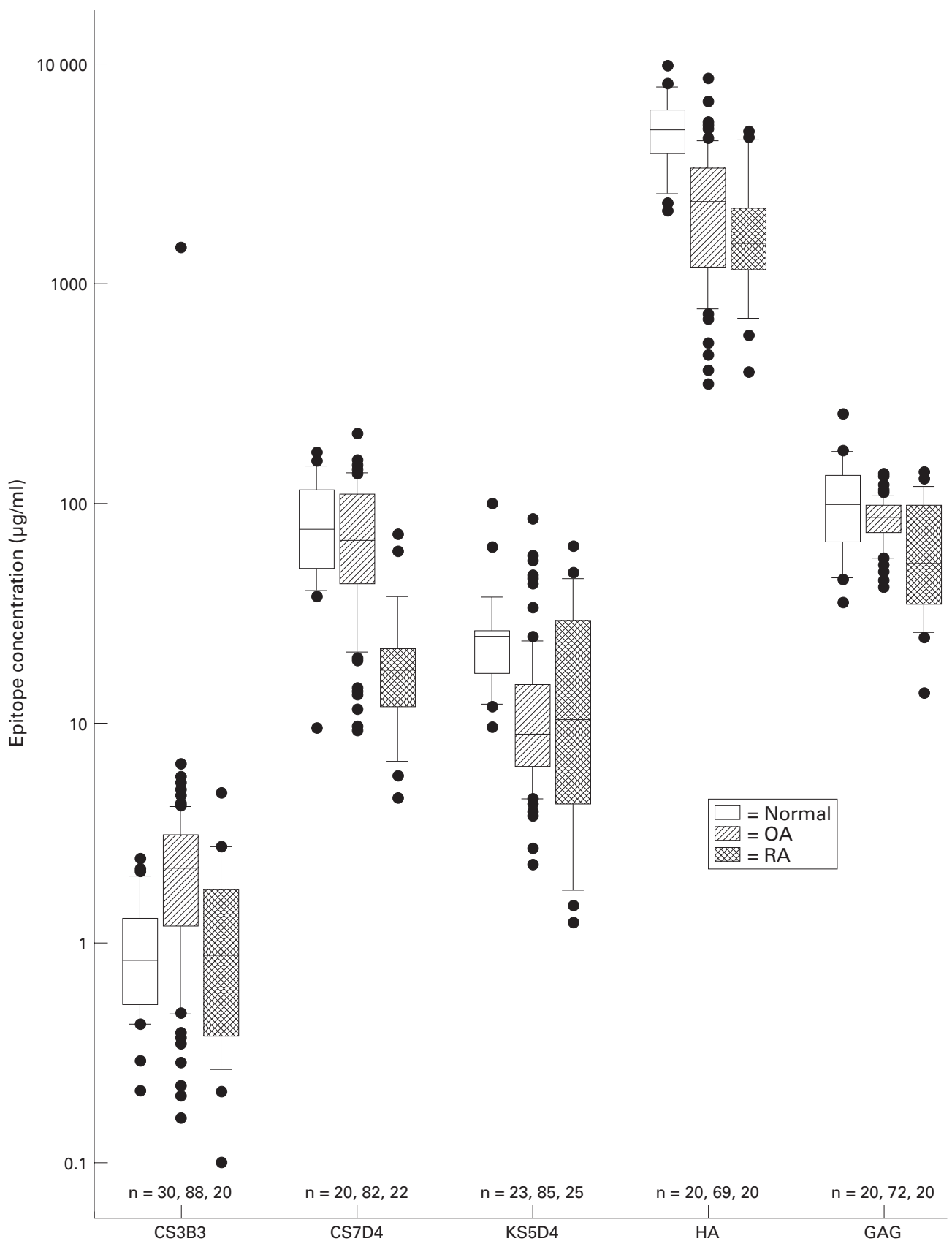

Figure 2 Comparison between $R A, O A$, and normal knees for all epitopes measured. The central solid line represents the median, with the box representing the middle $50 \%$ of the data. The error bar cap lines mark the 10th and 90th percentiles. Dots represent individual data points outside the 10th and 90th percentiles.

COMPARISON BETWEEN SUBSETS OF OA 3-B-3 concentrations were significantly lower in CPA compared with NGOA or LJOA (both $\mathrm{p}<0.01$; fig 4 ) but no differences were seen between subsets for 7-D-4, 5-D-4, HA or GAG concentrations. The 3-B-3/GAG ratio was significantly lower in CPA compared with NGOA or LJOA (both $\mathrm{p}<0.05$; fig 5) but no significant differences were seen between subsets for $7 \mathrm{D} 4 /$ GAG or 5D4/GAG.

EFFECT OF CLINICALLY ASSESSED INFLAMMATION 7-D-4 levels were reduced in 'active' compared with 'inactive' RA and in 'active' compared with 'inactive' OA (both $\mathrm{p}<0.05$ ). No differences between 'active' and 'inactive' knees were found for 7D4/GAG, 3B3,
3B3/GAG, 5D4, 5D4/GAG, HA or GAG in $\mathrm{RA}$, in (total) OA, or within subsets of OA (data not shown).

CORRELATION BETWEEN ELISA RESULTS AND $X$ RAY SCORES

No significant correlations were seen between any of the SF variables (concentrations or ratios) and the summated score for narrowing, the summated score for osteophyte, or the global joint score in OA patients (data not shown).

\section{Discussion}

This is the first large cross sectional study of SF CS and KS epitopes and HA to include (1) patients with well characterised OA, subdivided into clinical subsets, and (2) a broad age 


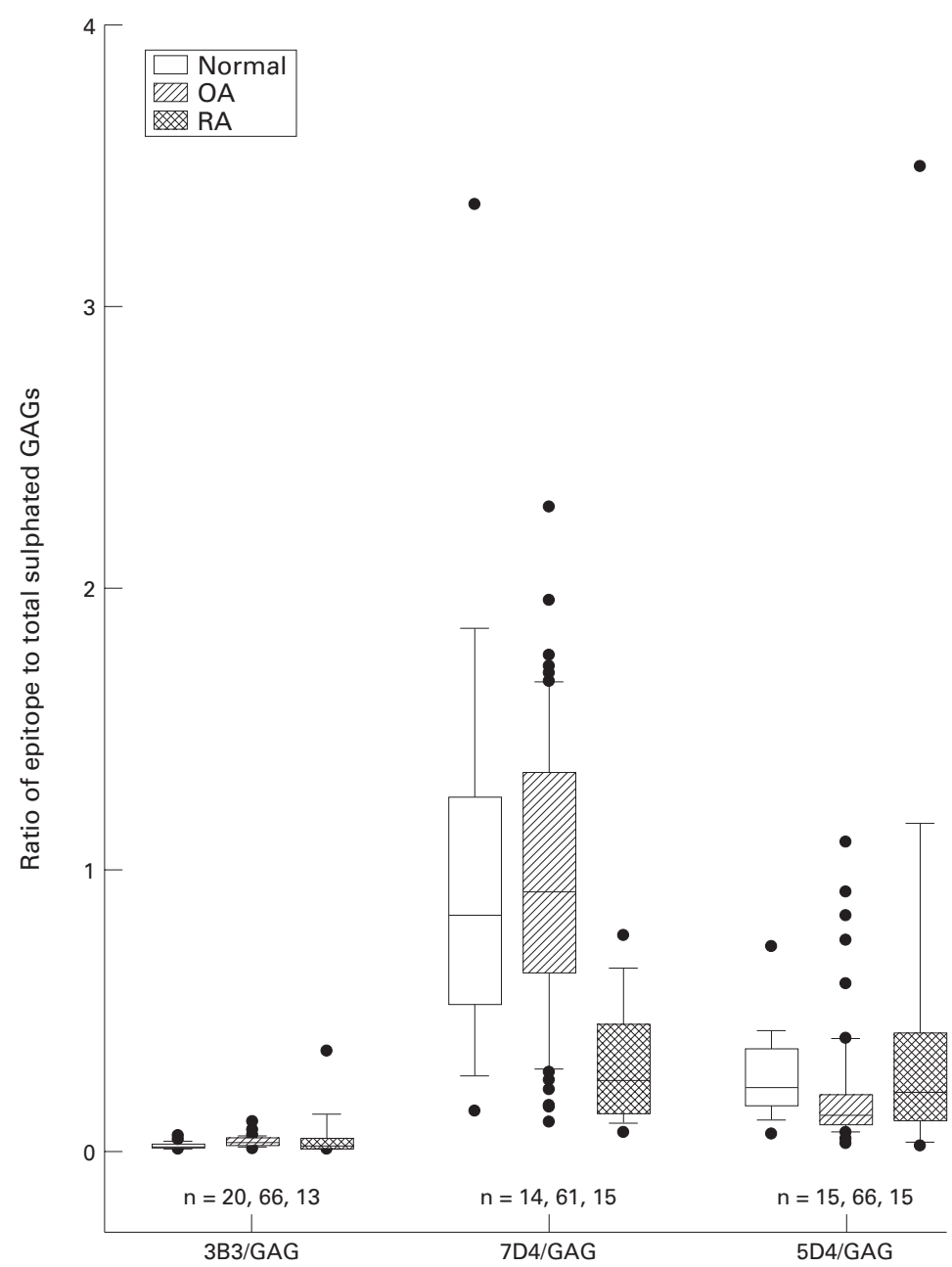

Figure 3 Comparison between $R A, O A$, and normal knees for ratio of chondroitin sulphate and keratan sulphate epitopes to total sulphated glycosaminoglycans. The central solid line represents the median, with the box representing the middle 50\% of the data. The error bar cap lines mark the 10th and 90th percentiles. Dots represent individual data points outside the 10th and 90 th percentiles.

range of normal volunteers with no history of knee trauma. The CS epitopes 3-B-3 and 7-D-4 have previously been shown to be increased in animal models of $\mathrm{OA}^{101120}$ and in human OA cartilage. ${ }^{12}$ Acute internal derangement of the knee may result in an increase in SF 7-D-4 and concomitant fall in SF 5-D-4 compared with the contralateral uninjured knee. ${ }^{13}$ However, large SF studies examining 3-B-3, 7-D-4 and 5-D-4 in human knee OA and its subsets, and in normal subjects over an age range of 21-83 years, have not been reported.

The correlation between right and left knees for all SF variables in subjects with bilateral fluids is of considerable interest. We found similar correlations for paired knee samples in a prospective study showing that estimation of these same SF variables at time of hospital referral does not predict subsequent radiographic progression of knee OA over two years. ${ }^{24}$ Campion et $a \bar{l}$ also noted correlation between right and left knees for SF 5-D-4 in the four patients with bilateral samples, though the problem of how to analyse groups containing more knees than contributing subjects was not answered. In a recent study of 202 OA knees from 134 patients strong correlation in SF pyrophosphate levels was found between knees of the same person ${ }^{25}$ and, as in the present and related ${ }^{24}$ study, only one knee per person was subsequently included in between group analyses. ${ }^{25}$ Alternative methods of analysing data in this situation have recently been discussed. ${ }^{26}$ Importantly, the correlations seen in this study between knees of the same person, together with lack of correlation between any SF variable and radiographic severity, suggest that these SF measures may predominantly reflect more an individual (constitutional, systemic) characteristic than the local extent of joint disease. However, because there was also correlation between knees for $x$ ray scores, this remains an open question. Future study of patients with discordant $x$ ray scores is required to consider this question.

In studies of putative SF diagnostic markers the inclusion of normal controls is clearly important. In our normal control group (mean age 45 , range 21-83 years) SF 3-B-3 levels increased, while SF HA levels decreased with age, thus reinforcing the requirement for regression analysis to account for confounding age differences between diagnostic groups. Using such analysis the CS epitope 3-B-3, both as a concentration and as a ratio to total GAG, was significantly increased in OA SF compared with normal SF. This is consistent with previous data on canine experimental $\mathrm{OA}^{101120}$ and human $\mathrm{OA}^{12}$ showing increased expression of 3-B-3 in OA cartilage, particularly in the most superficial layers where proteoglycan loss is most evident. ${ }^{12}$ These dog and human OA studies also report increases in 7-D-4, which was not observed in OA SF in our study. However, though both neo-epitopes associate with $\mathrm{OA}, 3-\mathrm{B}-3$ is less variable and more discriminative for abnormal human cartilage than 7-D-4. ${ }^{12}$ Patients in our study all had established, though not 'end stage', OA and it is possible that release of these epitopes into SF varies according to the stage and extent of cartilage attrition, as has been demonstrated for the quantity ${ }^{27}$ and type ${ }^{28}$ of proteoglycan fragments in RA SF. The increase in SF 3-B-3, and decrease in SF HA, with age in normal knees might be interpreted as a slow approach towards the OA situation in the older subjects, with physiological changes in cartilage that might predate radiographic or clinical evidence of OA. Although all normal subjects over age 50 had radiographically normal knees, more sensitive but invasive investigation (chondroscopy with cartilage biopsy) would be necessary to detect such mild 'preclinical' cartilage changes.

The observed reduction in SF 5-D-4 in OA might reflect synthesis of proteoglycans with lower $\mathrm{KS}$ content ${ }^{13}$ or qualitative alteration in KS structure. A similar decrease in SF KS (5-D-4) and accompanying increase in CS epitopes follows acute internal derangement of the knee, the greatest changes occurring within three months of injury and being greater for 7-D-4 than 3-B-3. ${ }^{13}$ Recently Sharif et $a l^{29}$ also reported lower SF 5-D-4 levels in OA compared with normal knees; unlike us they 


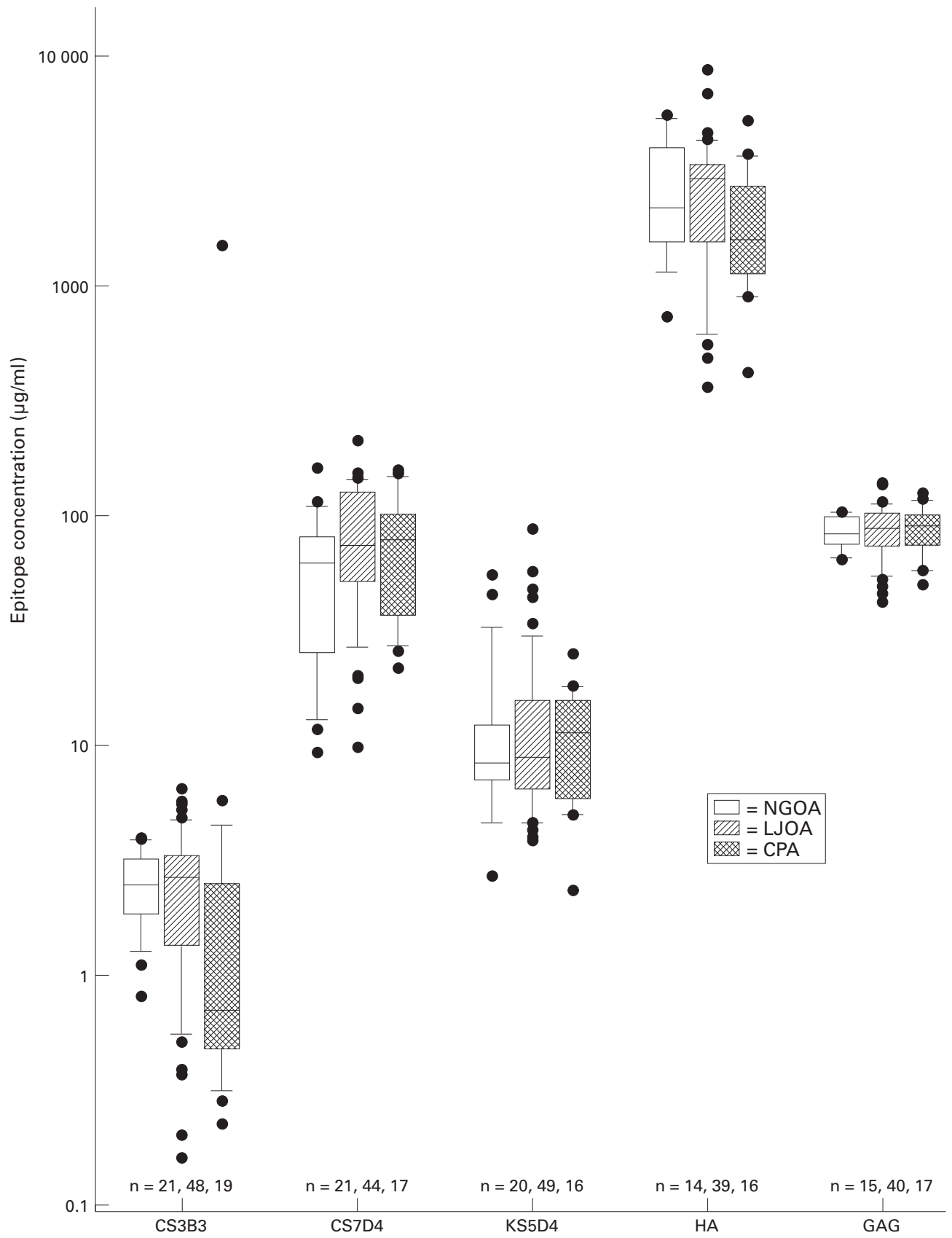

Figure 4 Comparison between the OA subclasses NGOA, LFOA, and CPA for all epitopes measured. The central solid line represents the median, with the box representing the middle $50 \%$ of the data. The error bar cap lines mark the 10th and $90 t h$ percentiles. Dots represent individual data points outside the 10th and 90th percentiles.

also found low SF 5-D-4 levels in RA knees and a negative correlation with age in 15 normal knees (from a group with mean (SD) age 40 (9) years). Radiographic assessments were not undertaken by Sharif et al, but in contrast with Campion et at we found no inverse correlation between SF 5-D-4 and degree of radiographic joint space loss. Campion et al, however, had no normal knee SF for comparison and designated knees as 'OA' even if radiographs were normal. The correlation they observed might therefore simply reflect normal $5-D-4$ levels in subjects with normal $x$ rays, and reduced 5-D-4 levels in those with radiographic narrowing (that is, definite $\mathrm{OA}$ ).

No increase in SF CS epitopes occurred in
7-D-4/GAG ratios, were reduced in RA compared with normal and OA knees. Such a finding supports the hypothesis that expression of these CS neo-epitopes reflects attempted repair with re-initiation of chondrocyte activity akin to that seen during development. ${ }^{13} \mathrm{RA}$ is characterised by atrophic cartilage and bone erosion; any reparative response, reflected by osteophyte and 'secondary OA', occurs late and is less impressive than in OA. ${ }^{30}$ Interestingly, the presence of clinically assessed knee 'inflammation' showed a negative association with SF 7-D-4 in both RA and OA, but no association with other SF variables. Study of the effects of cytokines on CS 7-D-4 expression and release might be of interest. The low HA concentrations in both OA and 


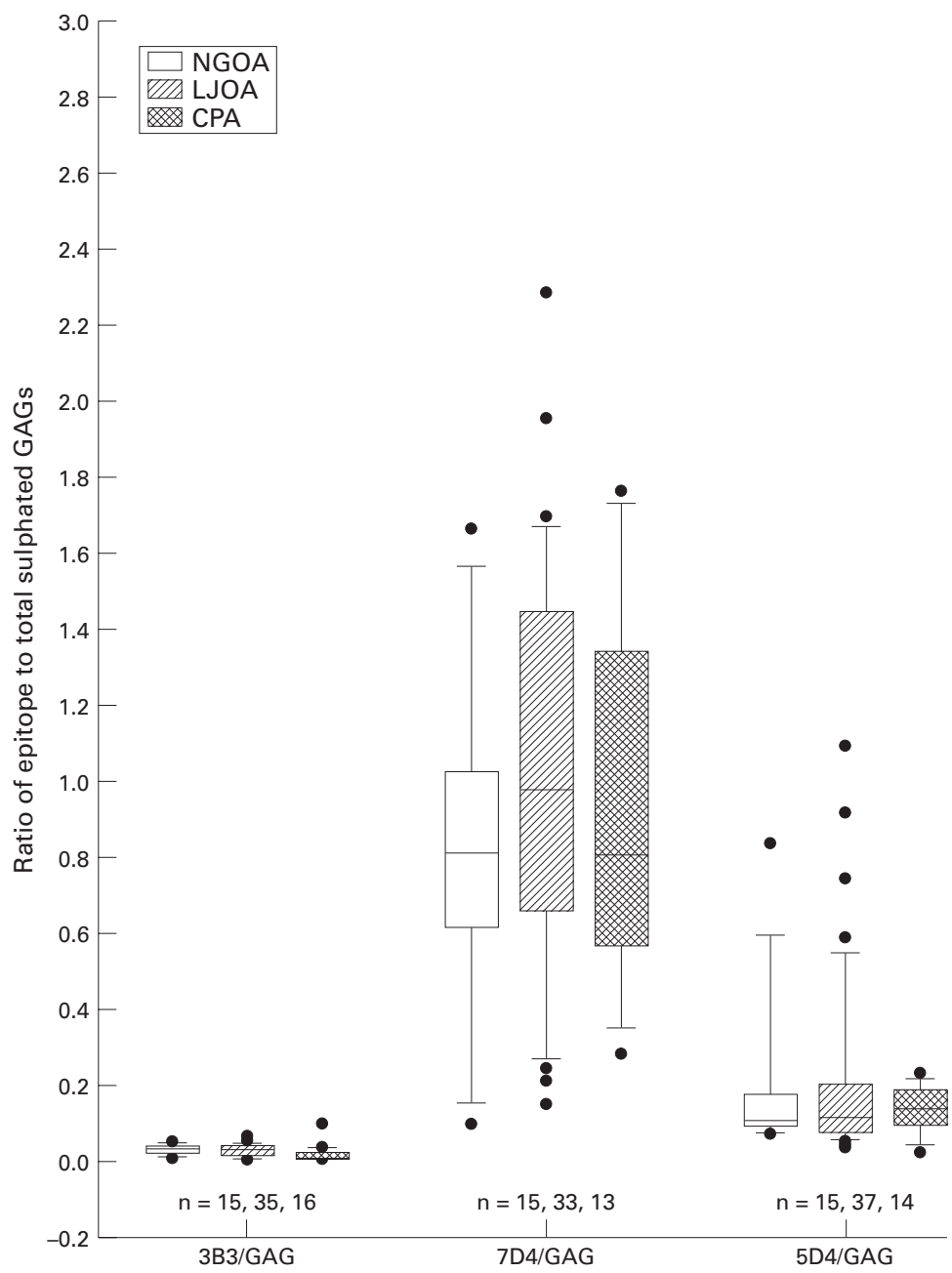

Figure 5 Comparison between the $O A$ subclasses $N G O A, L F O A$, and CPA for ratio of chondroitin sulphate and keratan sulphate epitopes to total sulphated glycosaminoglycans. The central solid line represents the median, with the box representing the middle $50 \%$ of the data. The error bar cap lines mark the 10th and 90th percentiles. Dots represent individual data points outside the 10th and 90th percentiles.

RA fluids accord with previous studies ${ }^{31} 32$ and with the clinical finding of reduced viscosity in abnormal, compared with normal, joint fluids. Total sulphated GAG SF levels have previously been reported to be similar in OA and RA, ${ }^{29} 3334$ either at the same concentrations found in normal knees, ${ }^{33}$ at lower concentrations than normal knee $\mathrm{SF}^{29}$ or higher than normal (compared with cadaveric knee $\mathrm{SF}^{35}$ ). In this study we found similar, reduced GAG concentrations in OA and RA compared with normal; we confirmed the previous observation ${ }^{34}$ of no change in GAG levels in OA or RA according to clinical inflammation. The discrepancies between reports presumably relates to differences in patient selection and characterisation, sample processing, GAG estimation, and source of control samples.

A single diagnostic category of ' $\mathrm{OA}$ ' is commonly used in laboratory based studies. It is therefore of interest to find greater increase in SF 3-B-3 in NGOA and LJOA compared with CPA, given the comparability between the three subgroups for age and radiographic knee OA scores. Using identical subgroup definitions we recently demonstrated differ- ences in knee SF for levels of plasminogen activators $^{36}$ and ratios of $6: 4$ sulphated CS disaccharides $^{37}$ in NGOA compared with LJOA or CPA. Such differences may support different pathogenesis or tissue response within OA subsets. Together with the other findings in this study it suggests that full assessment of the index joint (clinical inflammation, gross calcium crystal deposition, radiographic structural change) and of the person (Heberden's nodes, polyarticular versus oligoarticular OA) should be considered in future SF studies.

This study has several important caveats. We investigated only hospital based patients with established, relatively severe disease, and undertook only a single time point evaluation in a cross sectional study design. Serial SF measurements within people, and inclusion of subjects with earlier disease and less severe structural change may give different findings. Data from this study, however, delineate in established $\mathrm{OA}$ and RA different abnormal profiles of SF CS and KS epitopes, and suggest that local joint inflammation and unknown differences between OA subsets may influence some of these SF abnormalities. Further evaluation of these mechanisms seems warranted.

This work was funded by the Arthritis and Rheumatism Council to whom we are indebted. We are grateful for expert statistical advice from Andrea Baxendale (medical statistician).

1 Hardingham TE, Bayliss MT. Proteoglycans of articular cartilage: changes in ageing and in joint disease. Semin Arthritis Rheum 1990;20(suppl 1):12-33.

2 Mehmet H, Scudder P, Tang PW, Hounsell EF, Caterson B, Feizi T. The antigenic determinants recognised by three monoclonal antibodies to keratan sulphate involve sulphated hepta- or larger oligosaccharides of the poly (N-acetyl-lactosamine) series. Eur J Biochem 1986; 157:385-91.

3 Thonar EJ-MA, Lenz ME, Klintworth GK, Caterson B, Pachman LM, Glickman P, et al. Quantification of keratan sulphate in blood as a marker of cartilage metabolism. Arthritis Rheum 1985;28:1367-76.

4 Mehraban F, Finegan CK, Moskowitz RW. Serum keratan sulphate. Quantitative and qualitative comparisons in inflammatory versus non-inflammatory arthritides. Arthritis Rheum 1991;34:383-92.

5 Campion GV, McCrae F, Schnitzer TJ, Lenz ME, Dieppe PA, Thonar EJ-MA. Levels of keratan sulphate in the serum and synovial fluid of patients with osteoarthritis of the knee. Arthritis Rheum 1991;34:1254-9.

6 Sweet MBE, Coelho A, Schnitzer TJ, Lenz ME, Jakim I, Keuttner KE, et al. Serum keratan sulfate levels in osteoarKeuttner KE, et al. Serum keratan sulfate levels in
thritis patients. Arthritis Rheum 1988;31:557-60.

7 Spector TD, Woodward L, Hall GM, Hammond A, Williams A, Butler MG, et al. Keratan sulphate in rheumatoid arthritis, osteoarthritis, and inflammatory diseases. Ann Rheum Dis 1992;51:1134-7.

8 Block JA, Schnitzer TJ, Andersson GBJ, Lenz ME, Jeffery $\mathrm{R}$, McNeill TW, et al. The effect of chemonucleolysis on serum keratan sulphate levels in humans. Arthritis Rheum 1989;32:100-4.

9 Sharif M, George E, Shepstone L, Knudson W, Thonar EJ-MA, Cushnaghan J, et al. Serum hyaluronic acid level as a predictor of disease progression in osteoarthritis of the knee. Arthritis Rheum 1995;38:760-7.

10 Caterson B, Mahmoodian F, Sorrell JM, Hardingham TE, Bayliss MT, Carney ST, et al. Modulation of native chondroitin sulphate structure in tissue development and in droitin sulphate structure in tissue
disease. J Cell Sci 1990;97:411-7.

11 Visco DM, Johnstone B, Hill MA, Jolly GA, Caterson B. Immunohistochemical analysis of $3-\mathrm{B}-3(-)$ and $7-\mathrm{D}-4$ epitope expression in canine osteoarthritis. Arthritis Rheum 1993;36:1718-25.

12 Slater RR, Bayliss MT, Lachiewicz PF, Visco DM, Caterson B. Monoclonal antibodies that detect biochemical markers of arthritis in humans. Arthritis Rheum 1995;38:655-9.

13 Hazell PK, Dent C, Fairclough JA, Bayliss MT, Hardingham TE. Changes in glycosaminoglycan epitope levels in knee joint fluid following injury. Arthritis Rheum 1995; 38:953-9.

14 Ledingham JM, Regan M, Jones A, Doherty M. Radiographic patterns and associations of knee osteoarthritis in a hospital referred population. Ann Rheum Dis 1993; 52:520-6.

15 Arnett FC, Edworthy SM, Bloch DA, McShane DJ, Fries $\mathrm{JF}$, Cooper NS, et al. The American Rheumatism Associa- 
tion 1987 revised criteria for the classification of rheumatoid arthritis. Arthritis Rheum 1988;31:315-24

16 Spector TD, Cooper C, Cushnaghan J, Hart DJ, Dieppe PA. A radiographic atlas of knee osteoarthritis. London: SpringerVerlag, 1992

17 Doherty M, Richards N, Hornby J, Powell R. Relationship between synovial fluid $\mathrm{C} 3$ degradation products and local joint inflammation in rheumatoid arthritis, osteoarthritis and crystal associated arthropathy. Ann Rheum Dis 1988; 47:190-7.

18 Jones A, Hopkinson N, Pattrick M, Berman P, Doherty M. Evaluation of a method for clinically assessing osteoarthritis of the knee. Ann Rheum Dis 1992;51:243-5.

19 Hamilton E, Pattrick M, Doherty M. Inorganic pyrophosphate, nucleoside triphosphate pyrophosphatase, and cartilage fragments in normal human synovial fluid. $\mathrm{Br} \mathrm{J}$ Rheumatol 1991;30:260-4.

20 Ratcliffe A, Shurety W, Caterson B. The quantitation of a native chondroitin sulphate epitope in synovial fluid native chondroitin sulphate epitope in synovial fluid lavages and articular cartilage from canine experimental osteoarthritis

21 Fosang AJ, Hey NJ, Carney SL, Hardingham TE. An ELISA plate based assay for hyaluronan using biotinylated G1 domain (HA-binding region). Matrix 1990;10:306-13.

22 Farndale RW, Sayers C, Barrett AJ. A direct photospectrophotometric microassay for sulfated glycosaminoglycan in cartilage cultures. Connect Tissue Res 1982;9:247-8.

23 Farndale RW, Buttle DJ, Barrett AJ. Improved quantitation and discrimination of sulphated glycosaminoglycans by use of dimethylmethylene blue. Biochim Biophys Acta 1986;883:173-7.

24 Fawthrop F, Yaqub R, Belcher C, Bayliss M, Ledingham J, Doherty M. Chondroitin and keratan sulphate epitopes, glycosaminoglycans and hyaluronan in progressive versus non-progressive osteoarthritis. Ann Rheum Dis 1997; 56:119-22

25 Doherty M, Belcher C, Regan M, Jones A, Ledingham J. Association between synovial fluid levels of inorganic pyrophosphate and short term radiographic outcome of pyrophosphate and short term radiographic outco
knee osteoarthritis. Ann Rheum Dis 1996;55:432-6.

26 Zhang Y, Glynn RJ, Felson DT. Musculoskeletal disease research: should we analyse the joint or the person? J Rheumatol 1996;23:1130-4.
27 Saxne T, Heinegard D, Wollheim FA, Pettersson H. Difference in cartilage proteoglycan level in synovial fluid in early rheumatoid arthritis and reactive arthritis. Lancet $1985 ;$; $127-8$.

28 Saxne T, Heinegard D. Synovial fluid analysis of two groups of proteoglycan epitopes distinguishes early and late cartilage lesions. Arthritis Rheum 1992;35:385-90.

29 Sharif M, Osborne DJ, Meadows K, Woodhouse SM, Colvin EM, Shepstone L, et al. The relevance of chondroitin and keratan sulphate markers in normal and arthritic synovial fluid. Br J Rheumatol 1996;35:951-7.

30 CS-Szabo G, Roughley PJ, Plaas AHK, Glant TT. Large and small proteoglycans of osteoarthritic and rheumatoid articular cartilage. Arthritis Rheum 1995;38:660-8.

31 Balazs EA, Watson D, Duff IF, Roseman S. Hyaluronic acid in synovial fluid. Molecular parameters of hyaluronic acid in normal and arthritic human fluids. Arthritis Rheum 1967;10:357-76.

32 Dahl LB, Dahl IMS, Engstrom-Laurent A, Granath K. Concentration and molecular weight of sodium hyaluronate in synovial fluid from patients with rheumatoid arthritis and other arthropathies. Ann Rheum Dis 1985;44:817-22.

33 Silverman B, Cawston TE, Page Thomas DP, Dingle JT, Hazleman BL. The sulphated glycosaminoglycan levels in synovial fluid aspirates in patients with acute and chronic joint disease. Br J Rheumatol 1990;29:340-4.

34 Ratcliffe A, Doherty M, Maini RN, Hardingham T. Increased concentrations of proteoglycan components in the synovial fluids of patients with acute but not chronic joint disease. Ann Rheum Dis 1988;47:826-32.

35 Bensouyad A, Hollander AP, Dularay B, Bedwell AE, Cooper RA, Hutton CW, et al. Concentrations of glycosaminoglycans in synovial fluids and their relation with immunological and inflammatory mediators in rheumatoid arhritis. Ann Rheum Dis 1990;49:301-7.

36 Belcher C, Fawthrop F, Bunning R, Doherty M. Plasminogen activators and their inhibitors in synovial fluids from normal, osteoarthritis, and rheumatoid arthritis knees. normal, osteoarthritis, and rhe

37 Lewis S, Crossman M, Doherty M, Bayliss M, Mason R. Characterisation by capillary electrophoresis of synovial fluid chondroitin sulphates in osteoarthritis. [Abstract]. $\mathrm{Br}$ J Rheumatol 1995;34 (suppl 1):54. 